\title{
Imaging Care Requirements: Use of Functional Neuroimaging to Predict Dementia Caregiver Burden
}

\author{
Angus M.J. Prosser ${ }^{a} \quad$ John H. Spreadbury ${ }^{a} \quad$ Livia Tossici-Bolt $^{b}$ \\ Christopher M. Kipps ${ }^{a, c}$ \\ a Faculty of Medicine, Collaboration for Leadership in Applied Health Research and Care \\ (NIHR CLAHRC) Wessex, University of Southampton, Southampton, UK; ${ }^{b}$ Department of \\ Medical Physics, University Hospital Southampton NHS Foundation Trust, Southampton, \\ UK; ' Wessex Neurological Centre, University Hospital Southampton NHS Foundation Trust, \\ Southampton, UK
}

\section{Keywords}

Dementia · Caregiver burden · Zarit Burden Interview · Single-photon emission computed tomography $\cdot$ Neuroimaging

\section{Abstract}

Background: Dementia caregivers frequently report high stress, with increased burden associated with worse outcomes for both patients and caregivers. Although many studies relate clinical phenotypes to burden, the relationship between imaging pathology and burden, irrespective of diagnosis, is unknown. This study investigated the relationship between caregiver burden and patient regional cerebral blood flow in dementia. Methods: Seventy-seven patients with cognitive impairment undergoing brain perfusion single-photon emission computed tomography imaging in normal clinical care and their caregivers were recruited. Caregiver burden was ranked from "little" to "severe" using the Zarit Burden Interview and perfusion values extracted from the patient images for predefined regions of interest. The associations between burden score and regional function on imaging were tested. Results: Burden score was significantly higher for caregivers of patients with abnormal perfusion compared to those with normal perfusion in the left and right frontal, right parietal, and right temporal lobes. No difference in burden was found in the left parietal or temporal groups. Correlations showed that a higher caregiver burden was associated with lower patient perfusion scores in the same regions. Conclusion: Caregiver burden is strongly related to the extent of frontal or right-predominant parietal or temporal lobe dysfunction. Regional abnormality on perfusion imaging can be used to facilitate identification of individuals who are likely to create a high burden on caregivers. 


\section{Introduction}

Caregiver burden refers to the adverse effects perceived or experienced in caring for another person and may include psychological or physical feelings of stress, pressure, or fatigue. Factors found to increase caregiver burden in dementia include behavioural disturbances and patient apathy [1-3], patient dysfunction in memory and recognising emotions [4], and dementia severity [5]. The dementia subtype of frontotemporal dementia (FTD), in particular the behavioural variant, is particularly linked with high caregiver burden $[5,6]$.

Identification of caregiver burden is important, with burden associated with depression and decreased quality of life for caregivers and with dementia patient institutionalisation [2, $7,8]$. If burden is identified, implementation of caregiver and patient interventions has the potential to reduce caregiver burden [9], improve caregiver depression [10,11], delay patient institutionalisation [12,13], and improve caregiver quality of life $[11,14]$.

The Zarit Burden Interview (ZBI) is considered a principal instrument in measuring caregiver burden in dementia [15]. The instrument includes items addressing the effects of caregiving on multidimensional aspects of the caregiver's life, including areas of quality of life, health, and eliciting negative thoughts/emotions. Several versions of the instrument are in use, all considered to possess sound psychometric properties of reliability and validity and suggesting an underlying two-factor structure reflecting "personal strain" and "role strain" [16].

Neuroimaging is commonly used to aid dementia diagnosis and provide information on dementia subtype. Perfusion single-photon emission computed tomography (SPECT) is a functional imaging technique that gives an indirect measure of brain function at rest that is commonly used to aid dementia diagnosis. Perfusion SPECT imaging gives good sensitivity and specificity for dementia prediction, with regional patterns of reduced function suggesting dementia subtype in addition to providing information on dementia severity and prognosis $[17,18]$. The direct correlation between regional deficits on perfusion SPECT imaging and presenting symptoms in an individual is well defined. Individuals with frontal regional deficits commonly present with behavioural, personality, or language changes, individuals with temporal deficits often show language or memory symptoms, and those with posterior deficits can show visuospatial processing and spatial mapping difficulties. However, the use of regional imaging to predict care needs and caregiver burden in a clinical setting is not well studied. The demonstration that abnormality within specific regions on imaging predicts caregiver burden, irrespective of dementia subtype or formal diagnosis, opens the way to better targeting of interventions for those at high risk of caregiver strain.

This study aimed to investigate the relationship between caregiver burden and patient regional functional deficits on perfusion imaging.

\section{Methods}

\section{Participants}

The participants involved in this study gave written informed consent to join the Research Ethics Committee-approved Brain Imaging in Dementia (BraIID) study. All patient participants from the BraIID study had reported cognitive decline at the time of recruitment and underwent perfusion hexamethylpropyleneamine oxime-SPECT (HMPAO-SPECT) imaging as part of the diagnostic process. The companions of patients were also recruited to provide information on patient and caregiver characteristics, including caregiver burden as assessed by the ZBI, clinical staging by the Clinical Dementia Rating (CDR) scale [19], behavioural assessment using the Cambridge Behavioural Inventory (CBI) [20, 21], and functional activity using the Functional Activities Questionnaire (FAQ) [22]. 
The data from 77 consecutive patients recruited to the BraIID study at the time of perfusion SPECT imaging, with a companion who knew them in a personal capacity, were analysed.

\section{Demographics and Neuropsychological Data}

Demographic information was collected from patient participants at the time of consent. Companions of patients completed the 22-item ZBI [23] and an informant-rated CDR, CBI, and FAQ within 2 weeks of patient scanning.

\section{Perfusion SPECT Imaging}

Perfusion SPECT imaging was completed in all cases using a ${ }^{99 \mathrm{~m}} \mathrm{Tc}$-HMPAO tracer. HMPAO scans were acquired on a GE Infinia 3/8 HK4 dual-head gamma camera equipped with lowenergy high-resolution collimators, following a circular orbit over $360^{\circ}, 180^{\circ}$ arc for each detector, with the radius minimised for each patient. The total number of projections was 120 , each acquired for $25 \mathrm{~s}$, with a $20 \%$ symmetrical energy window centred on the $140 \mathrm{keV}$ photopeak; the matrix size was $128 \times 128$ and the applied zoom was 1.33 , which resulted in a pixel size of $3.32 \mathrm{~mm}$. The images were checked for movement and reconstructed using a filtered back projection method with attenuation correction.

In preparation for statistical analysis, the HMPAO-SPECT reconstructed images were spatially normalised to a standard structural template and smoothed with a 16-mm kernel using Statistical Parametric Mapping 8 (SPM8) [24]. Cerebellar normalisation was also completed using in-house MATLAB code, correcting for age.

Regions of interest for the frontal, temporal, and parietal lobes were created using the WFU PickAtlas software and the Automated Anatomical Labelling digital atlas [25-27]. The Automated Anatomical Labelling digital atlas is set in the Montreal Neurological Institute space. Average normalised voxel value counts were obtained for each region of interest using the SPM8 MarsBaR toolbox for both patients and controls, giving a quantitative indication of perfusion [28]. Perfusion regions of interest for each individual were classified as normal or abnormal using a two standard deviation cut-off from 31 age-matched controls.

\section{Statistical Analysis}

The ZBI score was divided into those with little to no burden (score 0-20), mild to moderate burden (score 21-40), and moderate to severe and severe burden (score 41-88) as per recommended ZBI cut-offs. The moderate to severe and severe burden groups were combined due to the small number of individuals in the severe burden group $(n=3)$.

SPM8 voxel-based imaging comparisons comparing burden groups with controls to identify patterns of abnormally reduced perfusion were completed. Mann-Whitney U tests were used to compare mean ZBI scores across the same brain regions of interest. Correlation analysis compared ZBI total scores and perfusion values within regions of interest. Follow-up exploratory analyses using Spearman rho ( $p<0.01$ uncorrected threshold) tested for associations between specific ZBI items and regional perfusion values to identify whether specific patient regional deficits were associated with particular profiles of caregiver burden. The influence of age, patient and caregiver sex, CDR sum of boxes score, CBI total and domain score, and FAQ score on caregiver burden was also assessed using $\chi^{2}$ and Kruskal-Wallis testing. The CBI score for each domain and the total score were converted into a percentage of impairment: $0-25 \%$ was classified as mild, $26-50 \%$ as moderate, $51-75 \%$ as severe, and $>75 \%$ as very severe. This grading system was based on prior studies that have investigated the CBI tool $[21,29]$. 
Table 1. Participant demographics grouped by the ZBI

\begin{tabular}{|c|c|c|c|c|}
\hline & $\begin{array}{l}\text { Little to } \\
\text { no burden }\end{array}$ & $\begin{array}{l}\text { Mild to } \\
\text { moderate burden }\end{array}$ & $\begin{array}{l}\text { Moderate to } \\
\text { severe burden }\end{array}$ & $p$ value \\
\hline Total number & 31 & 32 & 14 & - \\
\hline Patients, male/female ratio & $16 / 15$ & $24 / 8$ & $10 / 4$ & ns \\
\hline Companions, male/female ratio & $12 / 19$ & $7 / 25$ & $3 / 11$ & ns \\
\hline Patient handedness, right/left/unknown & $29 / 1 / 1$ & $28 / 4 / 0$ & $12 / 0 / 2$ & ns \\
\hline Patient age, years (mean \pm SD) & $65.6 \pm 8.36$ & $72.2 \pm 8.25$ & $69.8 \pm 5.54$ & 0.006 \\
\hline Patient global CDR score, median (range) ${ }^{1}$ & $0.5(0-1)$ & $0.5(0.5-3)$ & $1.0(0.5-2)$ & 0.006 \\
\hline Patient FAQ score $(\text { mean } \pm \text { SD) })^{1}$ & $7.27 \pm 1.23$ & $12.0 \pm 1.09$ & $13.9 \pm 2.55$ & 0.005 \\
\hline
\end{tabular}

CDR, Clinical Dementia Rating; FAQ, Functional Activities Questionnaire; ns, not significant; SD, standard deviation; ZBI, Zarit Burden Interview. ${ }^{1}$ Total FAQ score: $n=71$; CDR score: $n=61$.

Table 2. Imaging region of interest $Z$ scores for each burden group when compared to perfusion SPECT controls

\begin{tabular}{|c|c|c|c|c|c|c|c|}
\hline & \multirow[t]{2}{*}{ Global } & \multicolumn{2}{|c|}{ Frontal } & \multicolumn{2}{|c|}{ Parietal } & \multicolumn{2}{|c|}{ Temporal } \\
\hline & & left & right & left & right & left & right \\
\hline Little to no burden & -0.92 & -0.85 & -0.92 & -1.10 & -1.02 & -0.92 & -0.74 \\
\hline Mild to moderate burden & -1.82 & -1.42 & -1.53 & -1.96 & -1.88 & -1.78 & -1.73 \\
\hline Moderate to severe burden & -2.10 & -2.18 & -2.42 & -1.98 & -2.20 & -1.55 & -1.84 \\
\hline
\end{tabular}

Burden grouping was completed using the Zarit Burden Interview. SPECT, single-photon emission computed tomography.

\section{Results}

\section{Participant Demographics}

The patient demographics are shown in Table 1. Caregivers in our sample were mostly female $(71 \%)$ and a spouse or partner of the patient with cognitive complaints $(80 \%)$, although some were siblings (8\%), adult children (8\%), and friends (4\%). Caregivers had a median ZBI score of 23 , ranging from 0 to 72 points out of 88 . There were slightly more male than female patients $(65 \%)$, with a median caregiver-rated CDR score of 0.5 and a median functional activity score of 9 out of 30. The mean age of patients was 69 years (standard deviation 8.34). The majority of patients were living with their spouse or partner (88\%) and were retired or not working due to illness (84\%), although a small percentage were still working at the time of recruitment (10\%; working status unknown in 6\%). Most individuals were right-handed (89\%), with a small number being left-handed (7\%; 4\% unknown). The burden score was not related to either caregiver or patient sex; however, burden groups differed significantly with respect to age $\left(\chi^{2}[2, n=77]=10.33, p=0.006\right)$. On average, those with none to little burden were younger than those with mild to moderate or with moderate to severe burden. Age, however, did not appear to influence perfusion imaging group status (normal versus abnormal).

\section{Associations between Caregiver Burden Group and Patient Regional Abnormality}

When comparing ZBI burden groups against controls on a voxel by voxel basis using SPM8 (Fig. 1), burden score was shown to be strongly associated with global brain dysfunction, with patient global perfusion decreasing with increasing caregiver burden (Table 2). In 


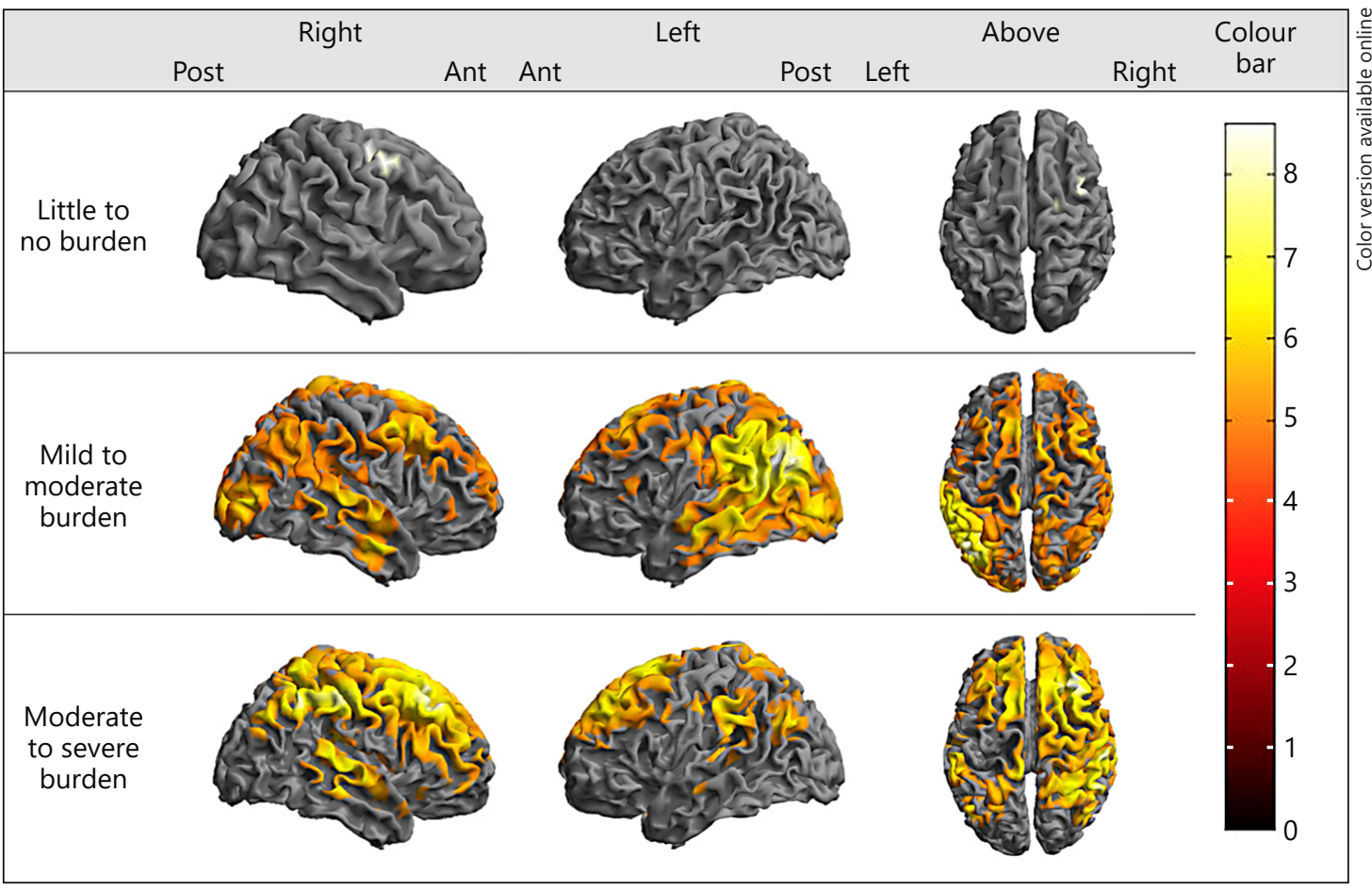

Fig. 1. SPM8 $t$-maps showing areas of reduced perfusion (heat scale) in individuals with little to none, mild to moderate, and moderate to severe burden as measured by the Zarit Burden Interview when compared to controls ( $p<0.05$, family-wise error corrected). Ant, anterior; Post, posterior; SPM, Statistical Parametric Mapping.

addition, burden was also associated with specific regional brain dysfunction. The moderate to severe burden group showed a predominantly frontal and right hemisphere reduction in patient perfusion when compared to controls. Those with mild to moderate burden showed a main area of reduced patient function in the left temporoparietal region around the angular gyrus, with generalised reduced function across the rest of the brain.

A Mann-Whitney test comparing regions and burden indicated that the ZBI total score was higher for individuals with abnormal frontal lobe perfusion (left frontal: $\mathrm{U}=408.5, p<$ 0.05 ; right frontal: $\mathrm{U}=491.0, p<0.05$ ) than those with normal perfusion in these regions. The total ZBI score was worse in those with right parietal $(U=500.5, p<0.05)$ and right temporal $(\mathrm{U}=431.5, p<0.01)$ abnormal perfusion. No difference was seen in total ZBI scores between the abnormal and normal left hemisphere parietal and left hemisphere temporal region groups.

\section{Correlations between Burden Score and Perfusion Values}

Spearman rho correlations between ZBI total score and cortical regions showed negative correlations indicative of higher caregiver burden associated with lower patient perfusion scores. The ZBI total score correlated negatively and significantly with both left $\left(r_{\mathrm{s}}=-0.337\right.$, $p=0.003)$ and right $\left(r_{\mathrm{s}}=-0.369, p<0.001\right)$ frontal regions, left $\left(r_{\mathrm{s}}=-0.304, p=0.007\right)$ and right $\left(r_{\mathrm{s}}=-0.313, p=0.006\right)$ parietal regions, and right temporal $\left(r_{\mathrm{s}}=-0.336, p=0.003\right)$ region. The left temporal region was not significantly correlated $\left(r_{\mathrm{s}}=-0.217, p=0.058\right)$.

Exploratory correlation analysis between individual item-region correlations revealed both item generalisation and specificity (all $p<0.01$; see online suppl. Table 1, www. 
karger.com/doi/10.1159/000486479). Items that significantly correlated with perfusion scores across all regions included those measuring stress and impact on health. In contrast, items measuring patient demands, personal time constraints, privacy, and social life were specific to the frontal regions, with right-sided dominance. The frontal and right temporal regions also correlated significantly with embarrassment and dependency.

\section{Influencing Covariates}

The CDR global score, indicating dementia symptom severity, was significantly different between burden groups $(\mathrm{H}[2]=10.3, p=0.006)$, and CDR showed a moderate positive correlation to burden score $\left(r_{\mathrm{s}}=0.355, p<0.005\right)$. Partial rank correlations correcting for CDR in the relationship between burden and regional perfusion showed that significance remained for the right frontal $\left(r_{\mathrm{s}}=-0.273, p=0.035\right)$, left parietal $\left(r_{\mathrm{s}}=-0.283, p=0.028\right)$, right parietal $\left(r_{\mathrm{s}}=-0.317, p=0.014\right)$, and right temporal $\left(r_{\mathrm{s}}=-0.376, p=0.003\right)$ regions. Burden groups also differed on FAQ performance, a specific measure of a patient's dependence and social function (instrumental activities of daily living). As burden increased, FAQ worsened (H[2] = $10.0, p=0.007)$, with FAQ showing a moderate positive correlation to burden score $\left(r_{\mathrm{s}}=\right.$ $0.434, p<0.001$ ). This appeared to be driven by the right frontal region only, with a higher FAQ seen in those with reduced perfusion in this region $(p=0.037)$. Partial rank correlations between burden and regions correcting for FAQ showed that weak significant correlations remained between burden and right frontal $\left(r_{\mathrm{s}}=-0.250, p=0.037\right)$, right parietal $\left(r_{\mathrm{s}}=-0.248\right.$, $p=0.039)$, and right temporal $\left(r_{\mathrm{s}}=-0.251, p=0.036\right)$ regions.

Comparison of behavioural scores as measured by the CBI showed a significant relationship between total score and burden $\left(\chi^{2}[6, n=76]=24.70, p<0.001\right)$, with a higher behavioural severity score associated with a worse caregiver burden. When individual domain impairment groups were compared to burden groups, the motivation $\left(\chi^{2}[6, n=76]=\right.$ $26.16, p<0.001)$, skills $\left(\chi^{2}[6, n=77]=17.09, p<0.01\right)$, as well as mood and stereotypic and motor behaviour domains $\left(\chi^{2}[6, n=77]=16.721, p<0.01\right)$ showed strong associations with burden.

\section{Discussion}

In this study we demonstrated that regional perfusion values on patient HMPAO-SPECT scans were strongly predictive of caregiver burden, irrespective of diagnosis. We found that burden is not only associated with the global pathological load but also with the pattern of regional pathology seen on imaging (Fig. 1).

Caregiver burden was largely driven by patient frontal lobe dysfunction. Those with reduced left or right frontal perfusion had a significantly higher burden score than those with normal frontal perfusion. This is likely due to patient behavioural or personality changes (neuropsychiatric symptoms), with higher caregiver burden associated with worse patient behavioural scores. Such neuropsychiatric features are well documented for behavioural-variant FTD, with patients frequently showing disinhibition, selfishness, lack of insightfulness, loss of embarrassment, irritability, and aggression [30]. Of note, however, is that we did not stratify the analysis by diagnosis but analysed burden simply in relation to regional pathology. A relationship between frontal pathology and neuropsychiatric symptoms is also present in other dementia subtypes, including Alzheimer disease [31-33]. Behavioural symptoms are frequently identified as a significant contributor to caregiver burden [34, 35].

Greater caregiver burden was also predicted by dysfunction of the right parietal and temporal regions. The right hemisphere has been previously demonstrated to be involved in 
mediating social and emotional behaviour in a mixed clinical sample in both structural [3638] and functional imaging studies [39-41], with right-sided changes associated with delusions, disinhibition, irritability, elation, and sleep disorder [42].

Although any reduction in cortical function correlated with an increase in caregiver burden, post hoc ZBI item analysis suggested that frontal type perfusion abnormality, predominantly right-sided, may place burden on caregivers through high patient demands as well as reduced privacy and social life for the caregiver, in addition to increased caregiver time constraints. Those with either frontal or right temporal regional functional reduction may be predisposed to higher levels of stress by exhibiting embarrassing behaviour and engendering higher levels of dependency. This information could be used to better target caregiver interventions in order to prevent or reduce caregiver burden at the time of diagnosis, and not simply when problematic behaviours contribute to crisis.

It has previously been noted that the CDR score correlates strongly with caregiver burden, with increased dementia severity associated with higher caregiver burden scores [5]. Similar correlations between caregiver burden and patient functional activities of daily living have also been found [43]. Our results agree with these findings, with overall CDR and FAQ higher in those with greater caregiver burden. Patient dementia severity and function, however, had little influence on the relationship between caregiver burden and patient regional perfusion deficits, suggesting that the location of reduced function affects burden independently of patient disease stage.

Previous studies highlight the importance of distinguishing the diagnostic subtypes of dementia when investigating caregiver burden and reactions to problems [44]. Although clearly interrelated, our results suggest that caregiver burden is predicted by patient regional abnormality, independent of a confirmed dementia subtype diagnosis. While regional abnormality largely defines the subsequent dementia syndrome, symptoms in dementia reflect the location of the underlying pathology. Frontal features are typical of FTD but are largely underappreciated in Alzheimer disease, despite their potential to impact caregiver burden. Although caregivers of FTD patients generally experience more burden than those of Alzheimer disease patients [44], simply grouping patients by subtype to identify caregivers at risk of high burden might fail to identify individuals with Alzheimer disease who have frontal or right predominant imaging deficits that may potentially present with challenging or complicated behaviours. Neuropsychiatric symptoms are common in Alzheimer disease patients [45-47] as well as mild cognitive impairment groups [48-50] and also frequently seen in mixed type and vascular dementia [51-53].

There are potential limitations to this study. Caregiver burden is multifactorial, and we did not control for the full range of potential confounding variables. In particular, we did not have measures of caregiver depression, which has been shown to be a strong influencer of burden, particularly in adult children caregivers [54]. Other factors such as hours providing care and level of support provided by health services can also affect burden, but were not measured in this study. These factors can however be measured directly in individual cases, and our findings suggest an influence of region-specific changes that are independent of caregiver characteristics.

The use of perfusion SPECT imaging for diagnosis is well established, but there is further information available from scans that is scarcely used and which may be valuable for assessing care needs. Regional abnormality on perfusion SPECT imaging at the time of diagnosis can be used to identify individuals likely to place the highest burden on their caregivers. Our study contributes further to understanding by identifying a component of caregiver burden that is independent of caregiver characteristics, and emphasises those features of burden that are most influenced by underlying pathology. Burden is most affected by frontal or right predominant parietal or temporal lobe dysfunction, irrespective of dementia subtype. With multidi- 
mensional caregiver interventions being effective in delaying patient institutionalisation and improving caregiver quality of life, this knowledge can be used to intervene with targeted support for caregivers with the highest risk.

\section{Acknowledgements}

This research was funded by the National Institute for Health Research (NIHR) Collaboration for Leadership in Applied Health Research and Care Wessex at the University Hospital Southampton NHS Foundation Trust. The views expressed are those of the authors and not necessarily those of the NHS, the NIHR, or the Department of Health. This study was supported by the Faculty of Medicine and the Faculty of Health Sciences at the University of Southampton. A.M.J. Prosser, J.H. Spreadbury, and C.M. Kipps receive support from the NIHR Collaboration for Leadership in Applied Health Research and Care (CLAHRC) Wessex.

\section{Disclosure Statement}

The authors have no potential conflicts of interest to disclose.

\section{References}

1 Armstrong N, Schupf N, Grafman J, Huey ED: Caregiver burden in frontotemporal degeneration and corticobasal syndrome. Dement Geriatr Cogn Disord 2013;36:310-318.

-2 Gaugler JE, Kane RL, Kane RA, Newcomer R: Unmet care needs and key outcomes in dementia. J Am Geriatr Soc 2005;53:2098-2105.

3 Beeri MS, Werner P, Davidson M, Noy S: The cost of behavioral and psychological symptoms of dementia (BPSD) in community dwelling Alzheimer's disease patients. Int J Geriatr Psychiatry 2002;17:403-408.

-4 Miller LA, Mioshi E, Savage S, Lah S, Hodges JR, Piguet O: Identifying cognitive and demographic variables that contribute to carer burden in dementia. Dement Geriatr Cogn Disord 2013;36:43-49.

5 Mioshi E, Foxe D, Leslie F, Savage S, Hsieh S, Miller L, et al: The impact of dementia severity on caregiver burden in frontotemporal dementia and Alzheimer disease. Alzheimer Dis Assoc Disord 2013;27:68-73.

6 Riedijk SR, De Vugt ME, Duivenvoorden HJ, Niermeijer MF, Van Swieten JC, Verhey FRJ, et al: Caregiver burden, health-related quality of life and coping in dementia caregivers: a comparison of frontotemporal dementia and Alzheimer's disease. Dement Geriatr Cogn Disord 2006;22:405-412.

7 Schulz R, Boerner K, Shear K, Zhang S, Gitlin LN: Predictors of complicated grief among dementia caregivers: a prospective study of bereavement. Am J Geriatr Psychiatry 2006;14:650-658.

$>8$ Yaffe K, Fox P, Newcomer R, Sands L, Lindquist K, Dane K, et al: Patient and caregiver characteristics and nursing home placement in patients with dementia. J Am Med Assoc 2002;287:2090-2097.

-9 Gaugler JE, Roth DL, Haley WE, Mittelman MS: Can counseling and support reduce burden and depressive symptoms in caregivers of people with Alzheimer's disease during the transition to institutionalization? Results from the New York University Caregiver Intervention Study. J Am Geriatr Soc 2008;56:421-428.

10 Sörensen S, Pinquart M, Duberstein P: How effective are interventions with caregivers? An updated metaanalysis. Gerontologist 2002;42:356-372.

11 Leung P, Orgeta V, Orrell M: The effects on carer well-being of carer involvement in cognition-based interventions for people with dementia: a systematic review and meta-analysis. Int J Geriatr Psychiatry 2017;32:372385.

12 Lawton MP, Brody EM, Saperstein AR: A controlled study of respite service for caregivers of Alzheimer's patients. Gerontologist 1989;29:8-16.

-13 Mittelman MS, Ferris SH, Steinberg G, Shulman E, Mackell JA, Ambinder A, et al: An intervention that delays institutionalization of Alzheimer's disease patients: treatment of spouse-caregivers. Gerontologist 1993;33: 730-740.

14 Belle SH, Burgio L, Burns R, Coon D, Czaja SJ, Gallagher-Thompson D, et al: Enhancing the quality of life of dementia caregivers from different ethnic or racial groups: a randomized, controlled trial. Ann Intern Med 2006;145:727-738.

15 Bédard M, Molloy DW, Squire L, Dubois S, Lever JA, O’Donnell M: The Zarit Burden Interview: a new short version and screening version. Gerontologist 2001;41:652-657. 
16 Hébert R, Bravo G, Préville M: Reliability, validity and reference values of the Zarit Burden Interview for assessing informal caregivers of community-dwelling older persons with dementia. Can J Aging 2000;19: 494-507.

17 Devous MD: Functional brain imaging in the dementias: role in early detection, differential diagnosis, and longitudinal studies. Eur J Nucl Med Mol Imaging 2002;29:1685-1696.

18 Mortimer AM, Likeman M, Lewis TT: Neuroimaging in dementia: a practical guide. Pract Neurol 2013;13: 92-103.

19 Hughes CP, Berg L, Danziger WL: A new clinical scale for the staging of dementia. Br J Psychiatry 1982;140: 566-572.

20 Wedderburn C, Wear H, Brown J, Mason SJ, Barker RA, Hodges J, et al: The utility of the Cambridge Behavioural Inventory in neurodegenerative disease. J Neurol Neurosurg Psychiatry 2008;79:500-503.

21 Bozeat S, Gregory CA, Ralph MA, Hodges JR: Which neuropsychiatric and behavioural features distinguish frontal and temporal variants of frontotemporal dementia from Alzheimer's disease? J Neurol Neurosurg Psychiatry 2000;69:178-186.

22 Pfeffer RI, Kurosaki TT, Harrah CH, Chance JM, Filos S: Measurement of functional activities in older adults in the community. J Gerontol 1982;37:323-329.

23 Zarit S, Orr NK, Zarit JM: The Hidden Victims of Alzheimer's Disease: Families under Stress. New York, New York University Press, 1985.

24 Friston KJ: Statistical parametric mapping; in Thatcher RW, Hallett M, Zeffiro TA, John ER, Huerta M (eds): Functional Neuroimaging: Technical Foundations. San Diego, Academic Press, 1994, pp 79-93.

25 Maldjian JA, Laurienti PJ, Kraft RA, Burdette JH: An automated method for neuroanatomic and cytoarchitectonic atlas-based interrogation of fMRI data sets. Neuroimage 2003;19:1233-1239.

26 Maldjian JA, Laurienti PJ, Burdette JH: Precentral gyrus discrepancy in electronic versions of the Talairach atlas. Neuroimage 2004;21:450-455.

27 Tzourio-Mazoyer N, Landeau B, Papathanassiou D, Crivello F, Etard O, Delcroix N, et al: Automated anatomical labeling of activations in SPM using a macroscopic anatomical parcellation of the MNI MRI single-subject brain. Neuroimage 2002;15:273-289.

28 Brett M, Anton JL, Valabregue R, Poline JB: Region of interest analysis using an SPM toolbox. Neuroimage 2002; 16:abstract 497.

29 Lillo P, Mioshi E, Zoing MC, Kiernan MC, Hodges JR: How common are behavioural changes in amyotrophic lateral sclerosis? Amyotroph Lateral Scler 2011;12:45-51.

-30 Bathgate D, Snowden JS, Varma A, Blackshaw A, Neary D: Behaviour in frontotemporal dementia, Alzheimer's disease and vascular dementia. Acta Neurol Scand 2001;103:367-378.

-31 Tekin S, Mega MS, Masterman DM, Chow T, Garakian J, Vinters HV, et al: Orbitofrontal and anterior cingulate cortex neurofibrillary tangle burden is associated with agitation in Alzheimer disease. Ann Neurol 2001;49: $355-361$.

-32 Lyketsos CG, Carrillo MC, Ryan JM, Khachaturian AS, Trzepacz P, Amatniek J, et al: Neuropsychiatric symptoms in Alzheimer's disease. Alzheimers Dement 2012;7:532-539.

33 Bruen PD, McGeown WJ, Shanks MF, Venneri A: Neuroanatomical correlates of neuropsychiatric symptoms in Alzheimer's disease. Brain 2008;131:2455-2463.

-34 de Vugt ME, Stevens F, Aalten P, Lousberg R, Jaspers N, Winkens I, et al: Behavioural disturbances in dementia patients and quality of the marital relationship. Int J Geriatr Psychiatry 2003;18:149-154.

-35 Allegri RF, Sarasola D, Serrano CM, Taragano FE, Arizaga RL, Butman J, et al: Neuropsychiatric symptoms as a predictor of caregiver burden in Alzheimer's disease. Neuropsychiatr Dis Treat 2006;2:105-110.

-36 Rosen HJ, Allison SC, Schauer GF, Gorno-Tempini ML, Weiner MW, Miller BL: Neuroanatomical correlates of behavioural disorders in dementia. Brain 2005;128:2612-2625.

-37 Williams GB, Nestor PJ, Hodges JR: Neural correlates of semantic and behavioural deficits in frontotemporal dementia. Neuroimage 2005;24:1042-1051.

38 Thompson SA, Patterson K, Hodges JR: Left/right asymmetry of atrophy in semantic dementia: behavioralcognitive implications. Neurology 2003;61:1196-1203.

39 Davidson RJ, Irwin W: The functional neuroanatomy of emotion and affective style. Trends Cogn Sci 1999;3: 11-21.

40 Benoit M, Koulibaly PM, Migneco O, Darcourt J, Pringuey DJ, Robert PH: Brain perfusion in Alzheimer's disease with and without apathy: a SPECT study with statistical parametric mapping analysis. Psychiatry Res 2002; 114:103-111.

41 Ott BR, Noto RB, Fogel BS: Apathy and loss of insight in Alzheimer's disease: a SPECT imaging study. J Neuropsychiatry Clin Neurosci 1996;8:41-46.

-42 Boublay N,SchottAM, Krolak-Salmon P: Neuroimaging correlates of neuropsychiatric symptoms in Alzheimer's disease: a review of 20 years of research. Eur J Neurol 2016;23:1500-1509.

43 Razani J, Kakos B, Orieta-Barbalace C, Wong JT, Casas R, Lu P, et al: Predicting caregiver burden from daily functional abilities of patients with mild dementia. J Am Geriatr Soc 2007;55:1415-1420.

-44 de Vugt ME, Riedijk SR, Aalten P, Tibben A, van Swieten JC, Verhey FRJ: Impact of behavioural problems on spousal caregivers: a comparison between Alzheimer's disease and frontotemporal dementia. Dement Geriatr Cogn Disord 2006;22:35-41. 
45 Mega MS, Cummings JL, Fiorello T, Gornbein J: The spectrum of behavioral changes in Alzheimer's disease. Neurology 1996;46:130-135.

46 Haupt M, Kurz A, Jänner M: A 2-year follow-up of behavioural and psychological symptoms in Alzheimer's disease. Dement Geriatr Cogn Disord 2000;11:147-152.

47 Lyketsos CG, Lopez O, Jones B, Fitzpatrick AL, Breitner J, Dekosky S: Prevalence of neuropsychiatric symptoms in dementia and mild cognitive impairment: results from the cardiovascular health study. JAMA 2002;288: $1475-1483$.

48 Hwang TJ, Masterman DL, Ortiz F, Fairbanks LA, Cummings JL: Mild cognitive impairment is associated with characteristic neuropsychiatric symptoms. Alzheimer Dis Assoc Disord 2004;18:17-21.

49 Geda YE, Roberts RO, Knopman DS, Petersen RC, Christianson TJH, Pankratz VS, et al: Prevalence of neuropsychiatric symptoms in mild cognitive impairment and normal cognitive aging: population-based study. Arch Gen Psychiatry 2008;65:1193-1198.

50 Rosenberg PB, Mielke MM, Appleby B, Oh E, Leoutsakos J, Lyketsos CG: Neuropsychiatric symptoms in MCI subtypes: the importance of executive dysfunction. Int J Geriatr Psychiatry 2011;26:364-372.

51 Fuh JL, Wang SJ, Cummings JL: Neuropsychiatric profiles in patients with Alzheimer's disease and vascular dementia. J Neurol Neurosurg Psychiatry 2005;76:1337-1341.

52 Johnson DK, Watts AS, Chapin BA, Anderson R, Burns JM: Neuropsychiatric profiles in dementia. Alzheimer Dis Assoc Disord 2012;25:326-332.

53 Srikanth S, Nagaraja AV, Ratnavalli E: Neuropsychiatric symptoms in dementia-frequency, relationship to dementia severity and comparison in Alzheimer's disease, vascular dementia and frontotemporal dementia. J Neurol Sci 2005;236:43-48.

54 Kaizik C, Caga J, Camino J, O’Connor CM, McKinnon C, Oyebode JR, et al: Factors underpinning caregiver burden in frontotemporal dementia differ in spouses and their children. J Alzheimers Dis 2017;56:1109-1117. 